\title{
Modeling natural space ionizing radiation effects on external materials
}

\author{
Richur! L. Altiut" and Dabid L. Edwart, \\ ${ }^{a}$ ED44/Sverdrup Technology, Inc., MSFC Group, Huntsville AL 35806 \\ ${ }^{\mathrm{b}}$ ED31/Environmental Effects Group. NASA/MSFC, Huntsville AL 35812
}

\begin{abstract}

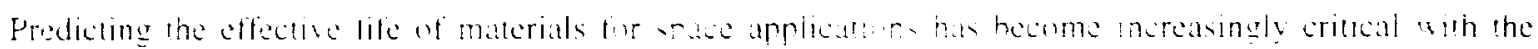

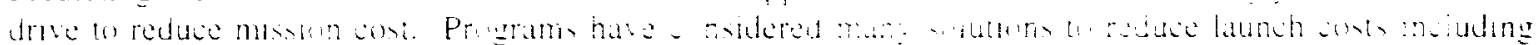
novel. low mass materials and than thermal blankets to reduce vadecratt mass. Determining the long-term survivability of these materials hefore launch is aritial for miston sucess. This presentation will describe an analysis performed on the suter layer of the passive thermal eontrol hlanket of the Huhble Space Telescope. This layer had degraded for unknusn reason during the mission. however annizing radiation (IR) induced embrittlement was suspected. A methodolong was developed which allowed direct comparison between the energy deposition of the natural envirnment and that of the laboratory generated

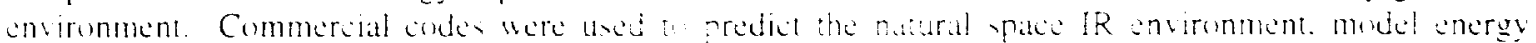

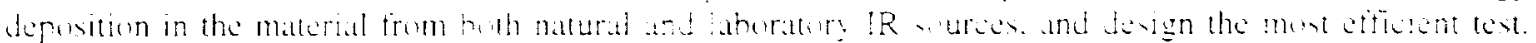
Results were optimised for total and local energ: deposition with an iterative preadsheet. This method has

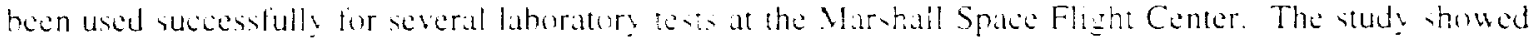

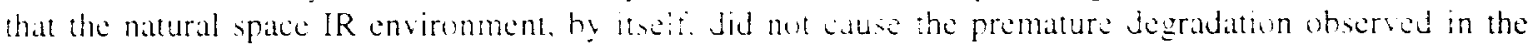
thermal blanket.
\end{abstract}

Keywords: Hubble Space Telescope, ionizing radiation. material degradation. multilayer insulation. Teflon

\subsection{I.NTRODUCTION}

The drive to reduce mission costs has resulted in innovative external materials for many applications in the space environment. Some of these materials hase no relevant flight experience and are designed with very low tolerance for degradation. Thermal control blankets. which can he reduced in thickness for a significant weight reduction, and mission specitic optical fïlms. which have little margin for degradation, are clear examples of potentially sensitive materials that are exposed (o) high ionizing radiation (IR) environments. The effect of the radiation environment, through mechanisms such as total ionizing duse (TID) and non-ionizing energy loss (NIEL), on sensitive materials like these impacts either the initial design or mission duration.

The passive multi-layer insulation (MLI) thermal control blanket degraded significantly during the first 10 vears of the Hubble Space Telescope's (HST) mission'. The MLI was inspected at 3.6 years and had developed minor cracks. After a total of 6.3 years on orbit. the blanket had developed severe cracks and was visibly degraded. Mechanical properties of samples returned to Goddard Space Flight Center (GSFC) were examined. This included tensile. bend. and micro-hardness tests. These tests indicated significant embrittement. loss of ultimate tensile strength. and an increase in surface hardness: effects commonly observed in radiation induced embrittlement. There were other several natural environment sources that had impacted the blanket surface and each of those environments was tested independently to determine if there had been an individual source of the observed damage. Suspect environments that were tested independently included natural space IR. ultosinlet light. solar X-rays. atomic oxygen. and therma! cycling.

Correspondence: Email: richurd.altslutt@mstic nisa gov: Telephone: (256) 544 8263: Fax: (256) 548807 
To determine the effects of the natural space IR environment on surface materials, the environment should be replicated as closely as possible in a laboratory experiment. The lahoratoy facility will be limited in the number or spectrum of energies avalahte while the spectrum of the natural spake environment to a continum of energies starting from less than a ket and continuing throgh hundreds of GeV. Since it will

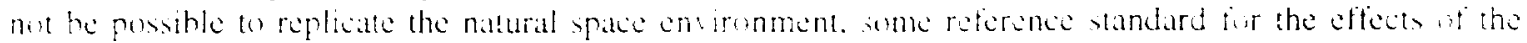
natural environment must be determined and the laboratory envirinnent will he generated to match the standard. The yardstick for comparison of the two environments was chosen to be total ionization energy deposition per unit mass (dose) as a function of depth in material. The units commonly used for dose are rads in material.

While many forms of radiation induced material damage are not related to ionizing energy deposition. the ionizing damage is usually two or more orders of magnitude higher than the non-ionizing damage ${ }^{2}$. This methodology could be used for non-ionizing dose from protons, but there are many additional code concerns with non-ionizing energy loss that were not considered here.

There are several commercial and freeware codes that can medel the natural space and lathoratory

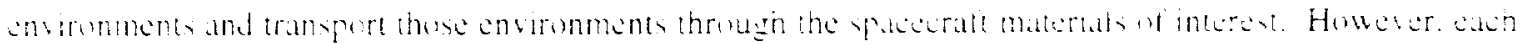
of these codes has signiticant limitations, so that there is not a single code capable of doing the entirety of this analysis. Each code is capable of performing some elements of the environmental analysis. By using the appropriate leatures of three separate codes and comparing their results using the reference standard. the complete analysis may be performed and veritied

Prediction of environment and initial dose as a function of depth was performed with the commercial code

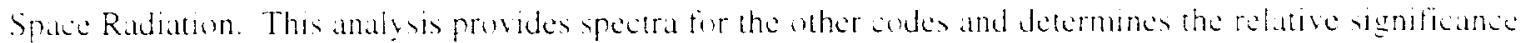

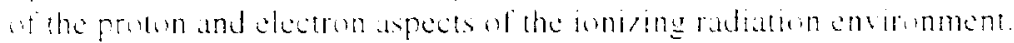

The Integrated TLGER Series (ITS) is a Monte Carlo style code produced and updated hy Oak Ridge National Lab. ITS was used to match the absorbed dose of electrons which enuld be produced in the laboratory. to the absorbed dose of the natural space trapped electron spectrum on 10 mils $(254 \mu \mathrm{m})$ of $\mathrm{FEP}$

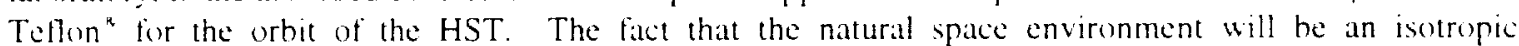
continuous spectrum. while the mono-energetic heams will be incident at $80^{\prime \prime}$ (near normal incidence) was considered along with the energy range limitations of the laboratory's electron heams.

The Stopping and Range of Ions in MatteriTransport of Radiation in Matter (SRIM 20OO/TRIM) codes are ion transport Monte Carlo codes designed and updated by IBM Research. The SRIM 2000 codes were used to determine the effects of protons on the material tested.

Space Radiation, using the internal code SHIELDOSE. was also used to verify the accuracy of ITS and SRIM 2000. SHIELDOSE has poor resolution in the low energy range, but it has significant laboratory data on the energy deposition of high-energy electrons and ions". A comparison case which can be performed on Space Radiation and ITS is a thin plate of Aluminum with an isotropic electron input. To compare TRIM and Space Radiation, the range of hydrogen ions in a flat plane of silicon was evaluated.

Marshall Space Flight Center's Environmental Effects Group pertiormed the tests. The laboratory used the iest design resulting from the modeling to approximate the absorbed dose of the natural space IR environment for missions of one to ten years. The effects of those tests on the material samples were determined by measuring the optical properties. GSFC also performed tensile, bending. and micro-hardness tests on the samples.

\subsection{INITIAL PARAMETERS FOR ORBIT, ENVIRONMENT, AND MATERIAL.}

Damage wo the MLI was assessed on a year-by-year basis for the 10-year reference mission for the HST with each vear assumed $(1)$ be equal to one lenth of the full mission. The approximate location of the HST wer the time the sample was exposed was a circular orbit at 611 kilometers and 28.5 " inclination. 
The high-energy protons will also penetrate ver: deeply into a material. The derage penetration depth for protons with energies greater than $10 \mathrm{MeV}$ is greater than $10 \mathrm{mil}$. From the plot of integral fluence in Figure 1. It can be estimated that less than $20 \%$ af the protons are of low enugh energy whe stopped in the material

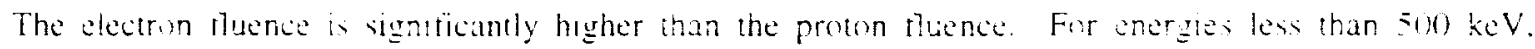
electrons have fluence more than 2 orders of magnitude greater than the protons. Because of these three factors, the electron fluence is the dominant source of the total dose in the first 200 mils of shielding.

The margin of uncertainty for the fluence predicted by the AE-8 and AP-8 models is traditionally considered to be a factor of two ${ }^{8-10}$. Actual fluence of particles may vary by as much as three orders of magnitude during short periods. The radiation fluence is also non-isotropic so that the facing or direction of travel can influence the dose received and the large structures. such as the inner and outer Van Allen helts. also vary with time and ipecific solar conditions. These properties are not well modeled at presint.

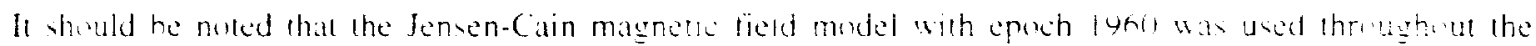

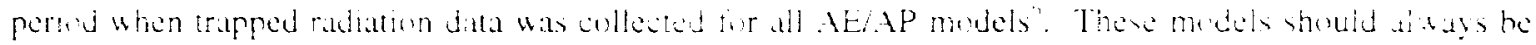
used with dates during the years in which data was taken. However the magnetic fields have shifted in the last to years, so the predictions will be at different orhital lecations than the spacecraft. There are versions of these codes being produced using current electron spectra. protun spectra. and mannetic field models. which will he good replacements.

The Environmental Effects Group - Space Environment Effects Team at Marshall Space Flight Center

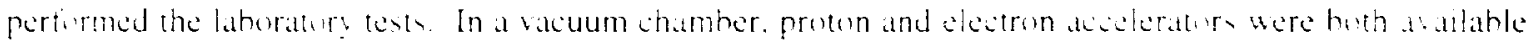

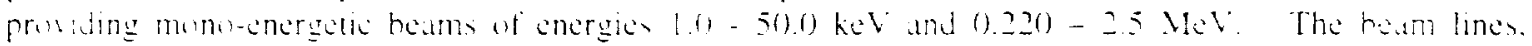
through which the radiation is emitted, are aligned at an inclination of 10 degrees from the normal to the eest vand. The heams produced by the laboratory hase a flux if appreximately $6.25 \times 10 "$ particke/em\%second with even coverage of the tert stand. which is square. three-inches on a side.

The passive heat shield for the HST is a 10 mil $(254 \mu \mathrm{m})$ layer of aluminized fluorinated ethylene propylene (FEP) Tetlon". The samples which were exposed in the laboratory were representative of the material used in orbit. The exact chemical composition and material preparation of this heat shield is not available. but the atomic composition and density is sufficient for radiation modeling. The material properties of Tetlon "were given as $72.66 \%$ fluorine. $22.97 \%$ carton. and $4.37 \%$ oxygen with a density of 2.15 grams/cm?

\subsection{COMPARISON OF TRANSPORT MODELS FOR RADIATION IN MATTER}

\subsection{Space Radiation with SHIELDOSE}

The commercial code Space Radiation uses the code SHIELDOSE to determine the energy deposition (dose) when transporting trapped proton and trapped electron spectra through a material. SHIELDOSE matches the ionizing radiation spectrum and target materials with pre-generated data". There are two difficulties with SHIELDOSE in the Space Radiation format. The first is that the code uses pre-generated data. si) that it doesn't contain the information to generate new energy deposition protiles for new materials. Second. the code was not written for energies less than $100 \mathrm{keV}$. The code assumes particles of $100 \mathrm{keV}$ are absorbed in the zone they are found, which creates an uncertainty of position on the scale of a micrometer. The stopping distances for higher energies are measured directly through experimentation. so that the error does not propagate throughout all of the results.

Space Radiation uses any continuous energy spectrum of protons or electrons in units of tluence/ $\mathrm{cm}^{2} / \mathrm{second}$. This is propagated through the designated material with a result in dose (rads in material as a function of depth in material integrated over mission duration. As Tetlon ${ }^{\text {* }}$ is not a material availatic in this patckage of Space Riadiation. the initial comparisons of the four spectrums were made using siticion. 
The solar cycle also has a large effect on the radiation environment. During solar maximum. the electrons are at their peak values and the protons are at the lowest. During solar minimum. protons are at their highest and electrons are at their lowest fluence.

While electron and proton eycles are opposite. the electrons are the usual driver for dose on external surfaces and protuns are more significant for shelded materials. Since the material was recovered during solar minimum. it had been present during the worst of the solar cycle for external surfaces, solar maximum. The cycle lasts approximately 11 years between peaks. The solar output remains at maximum for only about a year and a half, but the entire 7 year period around the peak is modeled as the maximum. For the mission's worst case radiation environment, only 3 of the 10 years are considered solar minimum.

The trapped radiation environment was modeled using the Space Radiation commercial package, which includes AE-8 and AP-8. The environment was cvaluated for the HST orbit using the AE-8 and AP-8 codes for electron and proton fluence respectively. These codes each have a version for solar maximum and solar minimum ${ }^{5}$. The results for one year on orbit for AE-8 min. AE-8 max. AP-8 min, and AP-8 max are presented in Figure 1. The ralactic cosmic radiation and soldr event environments, such as those that wecur during a coronal mass ejection or a solar proton event. were not included in the model recause their effects are several orders of magnitude less than the effects of trapped ionizing radiation inside the Van Allen Belts".

The proton spectrum has a significantly greater energy range than the electron spectrum. The high energy protons have a much greater penetration depth than the electrons. but they do not have a high linear energy transfer rate until they are either captured or their total energy drops below $100 \mathrm{keV}$. So while each of the high-energy protons has more energy to deposit in a material. it will spread that energy over a much larger range than the electrons. effectively reducing the amount of energy deposited in a thin film.

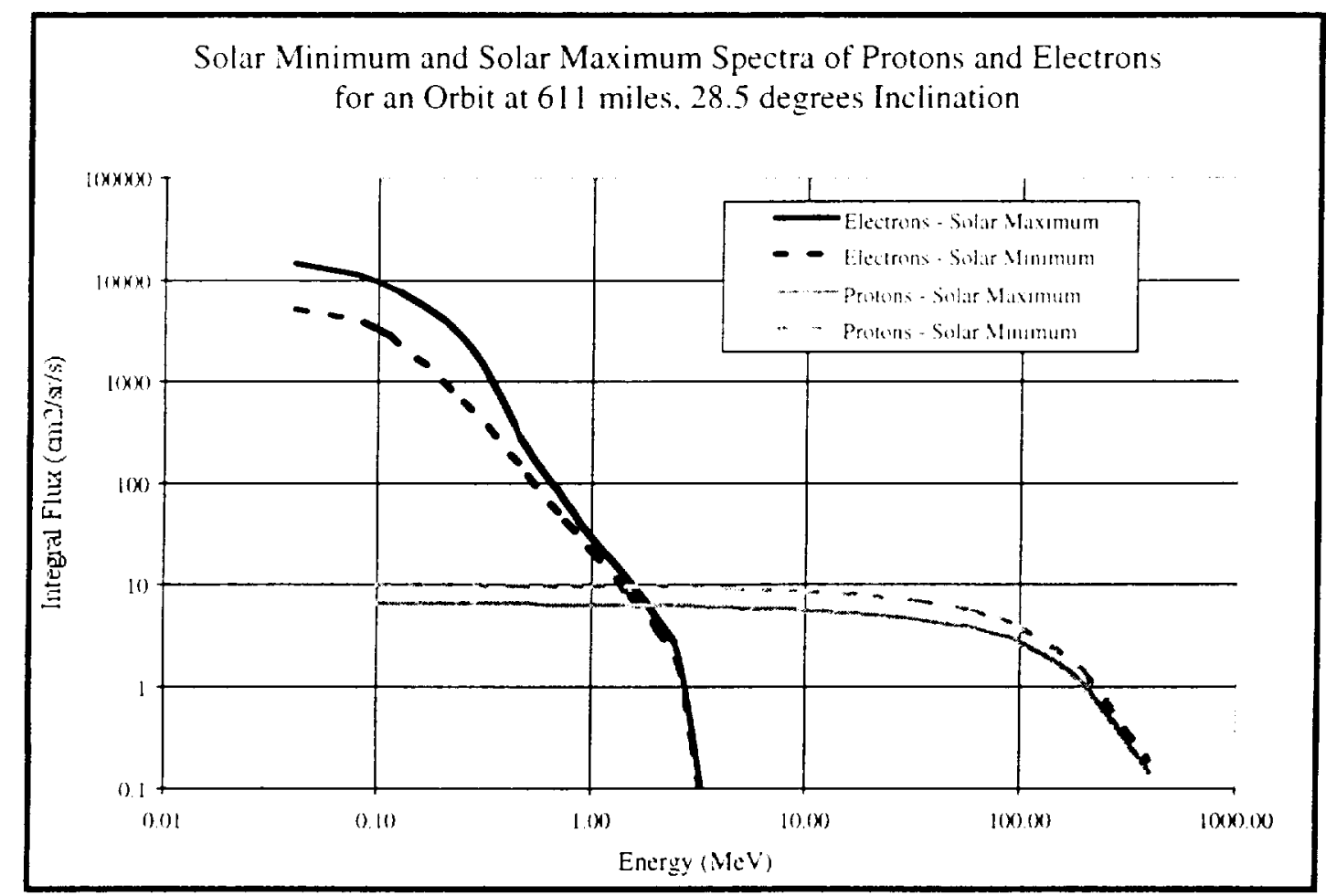

Figure 1. Trapped Proton and Electron Spectra for Solar Maximum and Solar Minimum Conditions. 
In Figure 2, the dose per unit depth for each of the four spectrums is presented. It is clear that the ionization caused by electrons is two orders of magnitude greater than the ionization caused by the protons in the first mil of the silicon and one order of magnitude throughout. More importantly. the electron dose has been shown to cause damage from as $10 \mathrm{w}$ as $10^{5}$ rads in stressed tluoro-polymers. such as Teflon ${ }^{\bar{n}}:=$. In other recent experimental work on polymers. the material effects of low energy clectrons. in high dowes have been clearly demonstrated ${ }^{13}$. Doses greater than $10^{\circ}$ rads have been found to cause material damage in some materials. but doses above $10^{7}$ rads cause material property degradation in most polymers 12 .

The proton dose, in comparison. is less significant by several orders of magnitude. Highiy stressed electronic circuits and optical devices may also be effected by doses of $10^{3}-10^{4}$ rads ${ }^{14}$, but no damage has been shown to polymers or other insulation materials in that range.

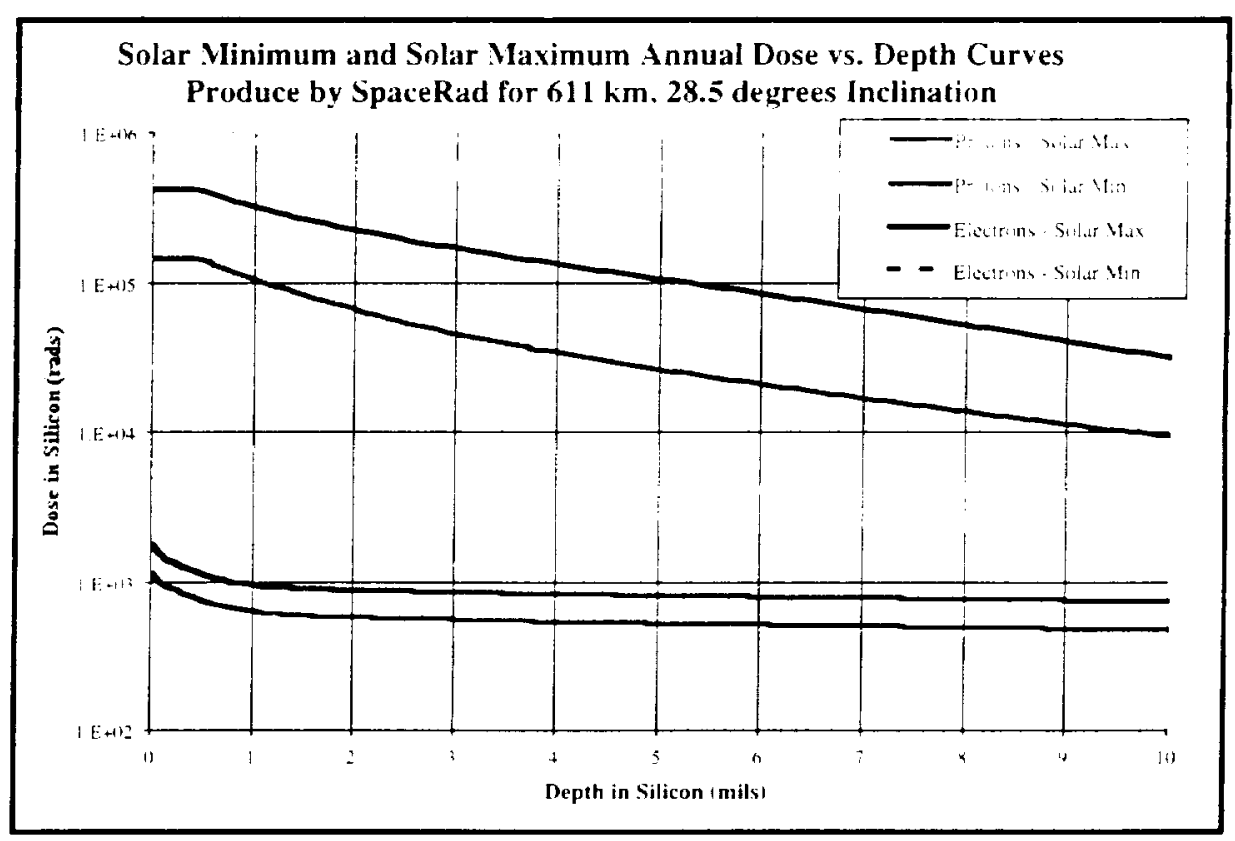

Figure 2. Dose as a Function of Depth Curves in Silicon for the Vatural Space Ionizing Radiation Environment tor One Year of Orbit tor the Hubble Space Telescope.

\subsection{Integrated TIGER Series}

The TIGER code was the Monte Carlo style code used to correlate the energy deposition results for trapped and laboratory electron environments. The electron spectrums presented in Figure 1 were used to represent the natural environment's isotropic spectrum. Single energy beams of the energies that could be produced in the laboratory at a $10^{\circ}$ inclination from the surface normal were also modeled to represent the possible laboratory beams. The sample of Teflon was divided into 100 regions in a thickness of 10 mils 10.0254 $\mathrm{cm}$ ) to measure energy deposition in each region. The low energy cut off, in ITS. was reduced to $1 \mathrm{keV}$ for greater accuracy when the step size was less than a few micrometers. This can be compared to SHIELDOSE. where the cut off cannot be adjusted and is set at $100 \mathrm{keV}$. For the regions very close to the surface, the low energy electrons will deposit the majority of the TID.

The results of each TIGER run are presented in the units of $\mathrm{MeV}-\mathrm{cm}^{2} /$ gram-particle. To analyze these numbers, the units are divided into two parts as in equation 1.

$$
\mathrm{MLV} / \mathrm{g}^{*} \mathrm{Cm}^{2} / \text { particle }
$$

The first can be converted into rads. 


$$
\begin{gathered}
100 \text { rads }=1 \text { Joule } / \mathrm{kg} \\
1 \mathrm{MeV}=1.6021 \times 10^{-1:} \text { Joules }
\end{gathered}
$$

so that the conversion factor can te found in equation $t$.

$$
1 \mathrm{MeV} / \mathrm{g}=1.6021 \times 10^{\circ} \mathrm{rads}
$$

Equation + was used to convert the results 6 rads $-\mathrm{cm}^{2} /$ particic.

The second part of the equation is the inverse of the fluence. electrons/em². so that the results can be converted to rads for a given exposure. The natural environment has an integrated fluence for mission duration. while the laboratory environment can adjust the flucnce produced so as to match to dose vs. depth curves of the natural environment. The worst case of the electron sectrum in dose in Tetlon vs. depth in Teflon ${ }^{2}$ is presented in Figure 3 with the factor of cur uncertinty presented as aror bars.

\section{Trapped Electron Dose vs. Depth in Teflon for 10 years at the Hubble Orbit}

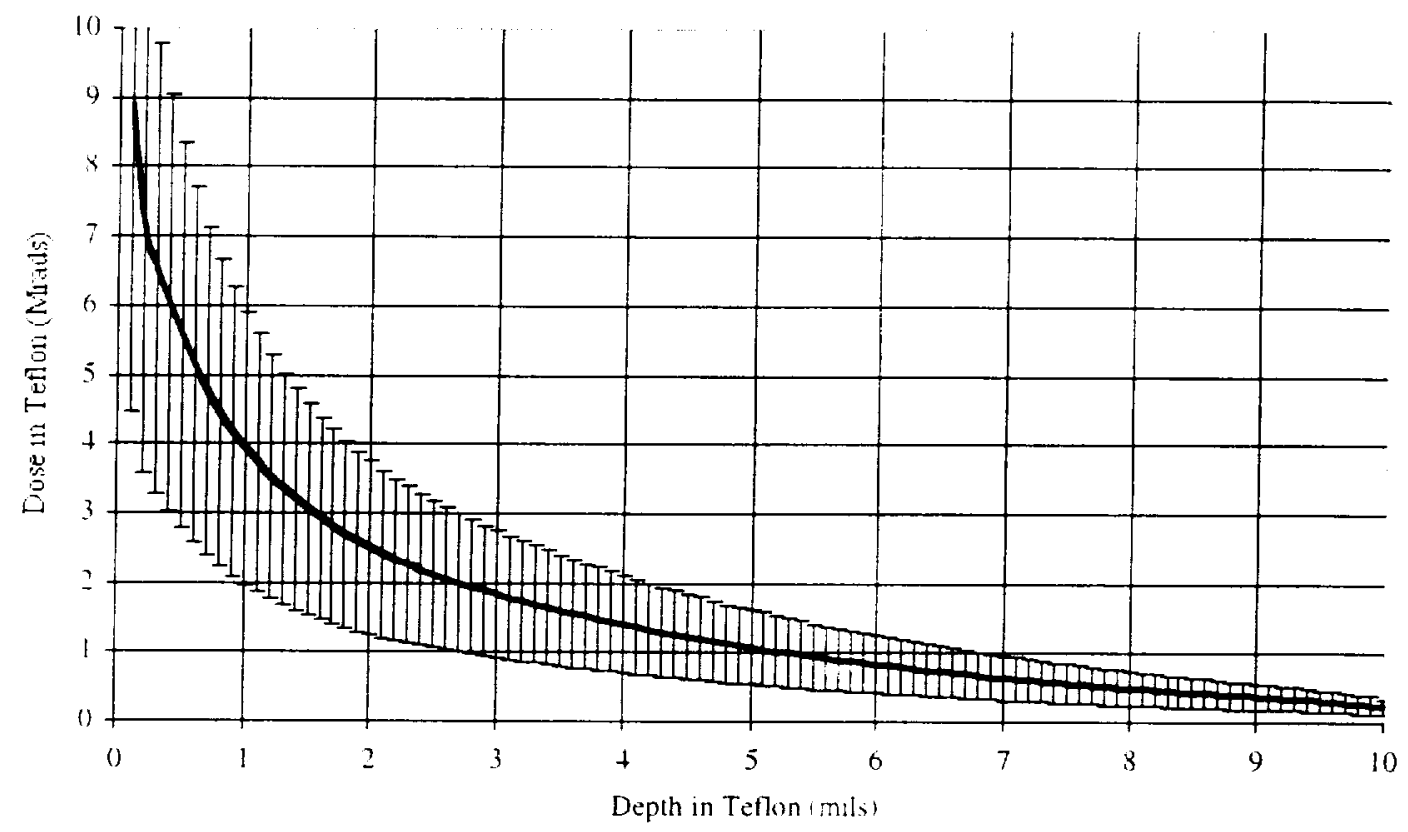

Figure 3. The Dose vs. Depth Curve for 10 Years at the Orbit of the Hubble Space Telescope as Predicted by the Integrated Tiger Series

The dose predicted in Figure 3 is significant for material properties. As stated previously. dose between 1 and 10 Mrads causes degradation in some tlouro-polymers. The prediction does not, however, determine that that amount of dose will cause an effect in this sample, only that there is a reasonable cause for further examination of a specific material in the laboratory.

\subsection{The Stopping and Range of Ions in Matter/Transport of Radiation in Matter (SRIM 2000/TRIM)}

The SRIM 2000 model is a pure. Monte Carlo style, ion propagation code't . The code transports an individual mono-energetic ion in a layered and angled material using probability and path length to determine the ion's energy loss to the material and the ion's total range. When the ion has stopped or passed through the material, the code repeats the process for the next ion. While an individual ion may produce 
any one of a number of energy loss interactions. the normalized sum of ten thousand ions usually converges to an accurate probability map. The number of juns used to generate the probability distribution can he adjusted to greater or lesser accuracy as recuiret. This process can be used 10 duplicate the laboratory environment. but cannot accurately reproduce the intropic, spread spectrum of the natural environment.

TRIM permits varying the ion species, energ: and angle of incidence of the particle beam source and multiple layers with variation in thekness and material composition for each layer in the target. The output may be graphically represented as Monte Carlo style path tracks such as shown in Figure 4, or area integrated energy deposition in units of ionization anergy per angstron depth as seen in Figure 5.

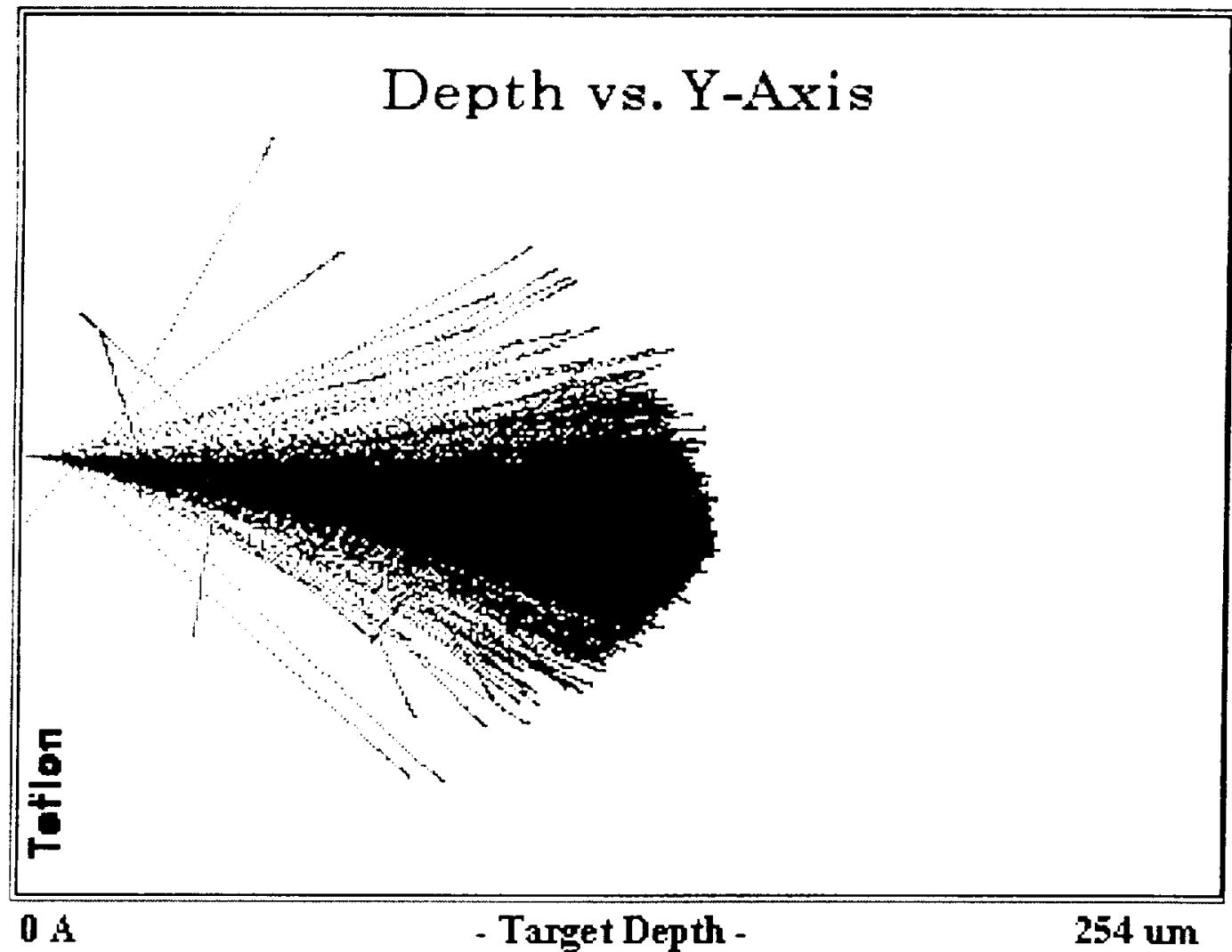

Figure 4. Monte Carlo Style Particle Traces Performed by TRIM Using a $4 \mathrm{MeV}$ Hydrogen Ion in 10 mils of Teflon

Figure 4 is a graphical representation of approximately $9.9 \times 10^{4}$ ion paths produced by TRIM in determining the normalized ionization and energy deposition profiles presented in Figure 5. The primary information obtained from Figure + is that there is very little divergence between the paths. This leads to the conclusion that the number of ion paths used was sufficient to guarantee convergence. When there are a large number of high probability events. more ions may be needed. It is also clear that even $4 \mathrm{MeV}$ ions did not penetrate the Teflon". As the lahoratory environment was limited to $2.5 \mathrm{MeV}$, a much higher energy beam device would have been required to accurately test this sample.

The results of the energy deposition profile are presented in units of eV/Angstrom/ion. To convert this to a unit of dose, the depth in material must be converted from angstroms, to units of mass, grams. This is done through a second step, converting the units of density from $\mathrm{g} / \mathrm{cm}^{3}$ to grams $/ \mathrm{cm}^{2}$-Angstrom. Then, given a specific integral fluence, in units of ions/ $\mathrm{cm}^{2}$. the units can be converted to dose as follows.

$$
\begin{aligned}
& \text { Normalized Energy Deposition (TRIM output) * Integral Fluence * } 1 / \mathrm{p}=\text { Energy Deposition/mass } \\
& \qquad \mathrm{eV} / \text { Angstrom/ion * ions/ } \mathrm{cm} 2 * 1 \mathrm{~cm}^{*} \text {-Angstrom)/grams * } 10^{-6} \mathrm{MeV} / \mathrm{eV}=\mathrm{MeV} / \mathrm{gram}
\end{aligned}
$$


The results of equation 5 can be converted, by equation 4 , into rads in material

Figure 5 compares the probability of energy transfer for each method for each ion. Direct ionization from the impinging ion clearly deposits the majority of the energy. Direct recoil is very efficient in this energy range, but it is not a very common event until the ion has lost most of its energy. Recoil, the primary source of non-ionizing energy loss. generates approximately 2 orders of magnitude less energy than direct ionization. The recoiled ions themsclves generate a small amount of ionization, roughly $10 \%$ of the total kinetic energy absorbed. Since Space Radiation has shown that the dose generated by protons should be approximately 1 kilorad per year. the non-ionizing dose should be approximately 10 rads/year. This is not a significant source of dose for material properties concerns.

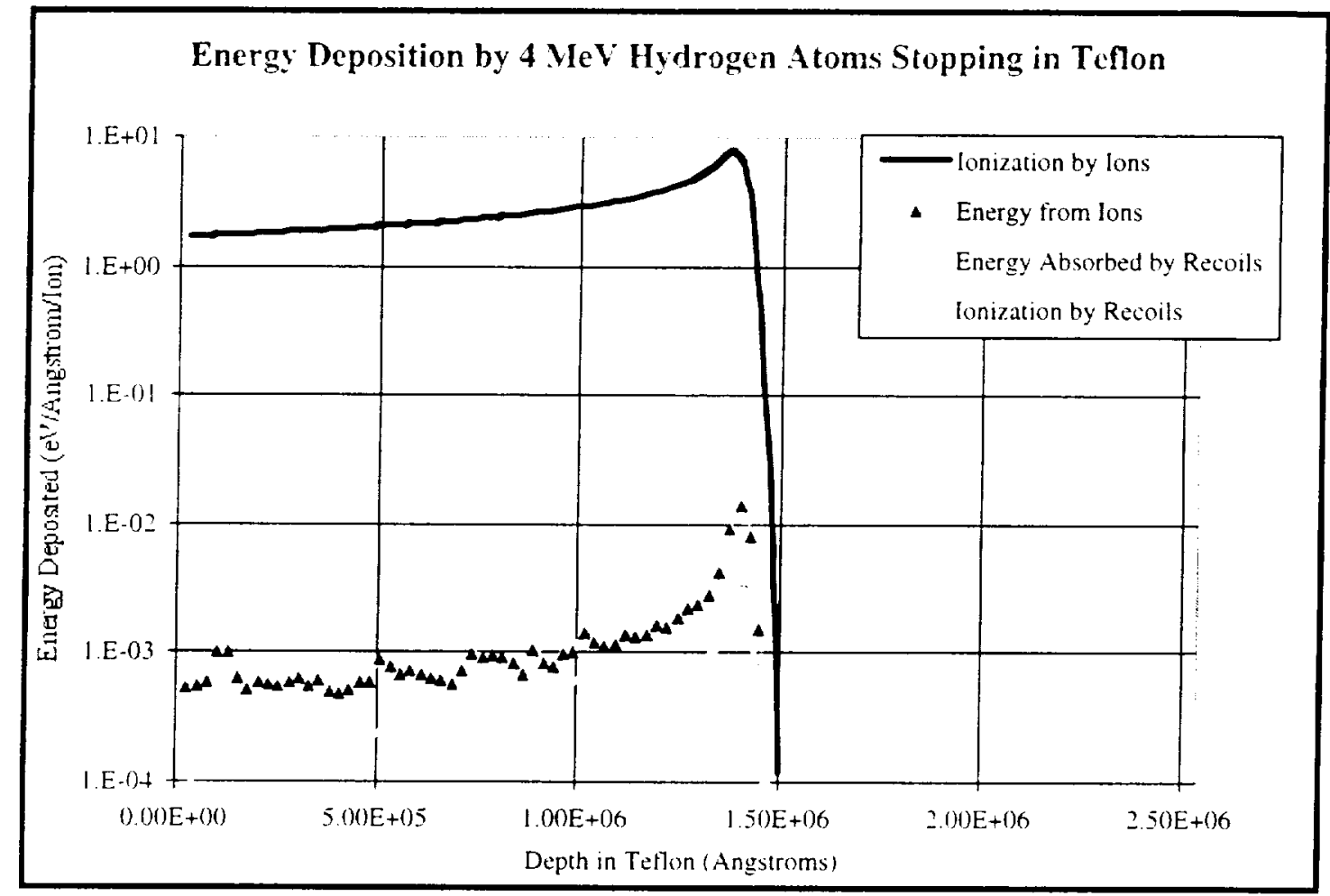

Figure 5. Normalized Energy Deposition Curves for Ionizing and Non-Ionizing Energy loss in Teflon ${ }^{B}$ for $4 \mathrm{MeV}$ Hydrogen Ions.

\subsection{Verification}

The codes were verified against each other by running comparison cases. A comparison that could be worked by both SHIELDOSE and TIGER was an isotropic exposure of a 10 mil thick aluminum plate to the solar minimum electron environment. The spectrum previously generated for solar minimum was used for inputs to both TIGER and SHIELDOSE. The comparison spectrum is presented in Figure 6 . The differences between the two results are obvious, but only a significant percentage of the result in the first mil $(25.4 \mu \mathrm{m})$. The greater energy deposition, in the first few mils, from the TIGER series code represents its more detailed analysis of the low energy electrons. At deeper penetrations, lower energy electrons have lesser effects so that the codes produce nearly equal results. 


\section{Comparison of Space Radiation and Tiger Series}

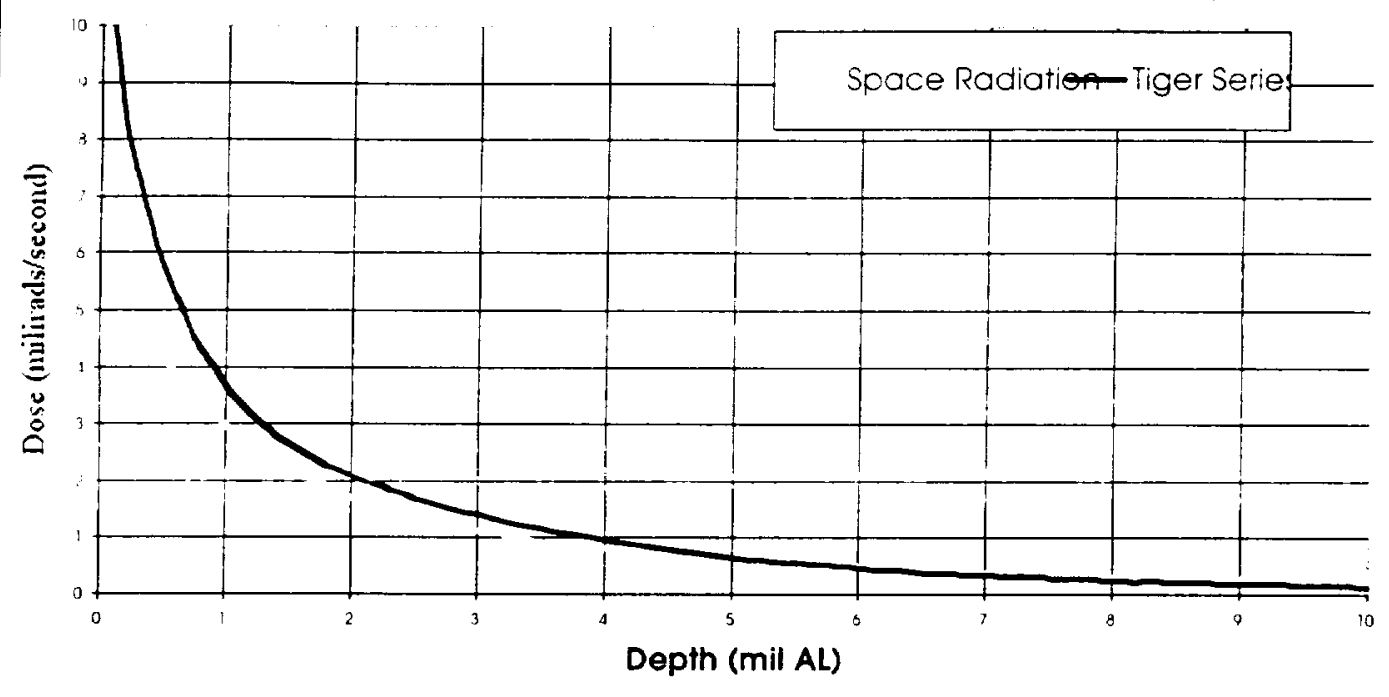

Figure 6. Comparison of Tiger Series Code against the Space Rad Code.

A comparison that could be produced by both SHIELDOSE and TRIM was the penetration of hydrogen ions in Silicon. TRIM was run with single energy beams at normal inclination to the surface, SHIELDOSE was run with a stepped spectrum from which peaks emerged on the dose vs. depth graph. The results are presented in Table 1.

\begin{tabular}{|c|c|c|}
\hline Energy & SHIELDOSE & TRIM \\
\hline $100 \mathrm{keV}$ & $<1$ micron & 0.8759 microns \\
\hline $400 \mathrm{keV}$ & 3.010 microns & 4.18 microns \\
\hline $600 \mathrm{keV}$ & 6.095 microns & 7.38 microns \\
\hline $1 \mathrm{MeV}$ & 13.72 microns & 13.4 microns \\
\hline
\end{tabular}

Table I. Penetration depth of protons in Silicon as predicted by SRIM and SHIELDOSE.

It is clear that SHIELDOSE and SRIM produce answers that differ on the order of a micron. However. as was seen in Figure 6, the values become less significant as the energy and distance into the material becomes larger. At 10 microns or $1 \mathrm{MeV}$ ions, the results are less than $3 \%$ different. These results are expected, SHIELDOSE was known to have this limitation, but Table 1 does show that the results from SHIELDOSE and SRIM are congruent at higher energies.

\subsection{RESULTS}

In Table 2 are the energies and total fluence used for matching the dose deposition of the beam and the natural space spectrum. For this result, the Chi square, $\chi^{2}$, was 0.36 , approximately a 1-sigma divergence in a comparison data set. The energy deposition by these beams is approximately $1.4 \%$ less than the deposition by the natural space environment over 10 years.

In Figure 9, the absorbed dose as a function of depth from the natural space IR spectra for the HST orbit is compared to the results from three of the electron beams. This is one example case of the combination of three beams. There are many variations in energy and fluence that could be used. By varying the initial choices in laboratory spectrum and different numbers of matching beams, the laboratory tests could be designed in several different ways. As there is a minimum time to turn the beams on and off of about one second, increased accuracy can be obtained by making the beam times as long as possible. The most 



\subsection{CONCLUSIONS}

The methodology developed in this paper made it possible to accurately compare the natural space IR environment to the laboratory environment. The accuracy was very close, even considering the limitations of the test facility. The initial work with Space Radiation showed that electrons were the prime drivers of surface dose in low Earth orbit. ITS was able to model both the natural space and laboratory environments satisfactorily. While SRIM 2000 shows that the protons did not produce a level of energy deposition sufficient to cause damage to materials, the methodology, closely related to that used with ITS. is still valuable for other projects. All three codes are shown to correlate closely, which validates the methodology developed here.

There are still areas where code improvements would be valuabie. For example. there is not a mudel for isotropic proton irradiation for very thin materials. However. this function can be added to TRIM with a table containing isotropic entry angles and smooth variations in energy. The table would have to be generated by a Fortran code for each specific environment, but by using Monte Carlo processes there should be no reduction in final accuracy. There is also difficulty in measuring neutron production from certain reactions, such as Aluminum's $(p, 2 n)$ interaction with protons. Nuclear reactions are not modeled by TRIM, but neutron codes may be used for some predictions in the future.

The laboratory tests showed no degradation of the sample for the ionizing radiation environment tested. This does not indicate that the electrons had no effect, but it does show that without contributory environmental or induced stresses, electrons were not responsible for the premature degradation observed on the HST MLI. The dose of electrons produced in this experiment was from $10^{6}$ to $10^{7}$ rads. The high surface dose caused by the low energy electrons may have had some effect, but there may not have been sufficient body damage to make a measurable optical or structural change. On the other hand, the electrons may not cause significant structural damage in Teflon ${ }^{8}$ until greater than $10^{8}$ rads. There may be no damage done until much higher dose levels unless the material is stressed.

There have been several tests with significantly higher doses, $10^{9}$ rads, which showed electrons degrading a thin material of similar structure ${ }^{12}$. However, the test performed on those materials included simultaneous stresses with protons and UV radiation. The combined effects of the three sources of material stress may have had a synergistic effect that enhanced degradation. More testing will be required to determine the rate of material properties degradation of Teflon from ionizing dose.

\section{ACKNOWLEDGEMENTS}

Financial support for this work was provided in part by NASA Contract NAS8-80436 to Sverdrup Technology, Inc.. under the Aerospace Environment Support Task $240-4400-0100$ in support of the ED44/Space Environment Team of the MSFC/Engineering Directorate.

\section{REFERENCES}

1. J. Dever. K. de Groh, J. Townsend, and L.L. Wang, Mechanical Properties Degradation of Teflon FEP Returned from the Hubble Space Telescope. NASA/TM-1998-206618, NASALeRC. January. 1998.

2. C.J. Marshall and P.W. Marshall, "Proton Effects and Test Issues for Satellite Designers", in the 1999 IEEE NSREC, IEEE Nuclear and Space Radiation Effects Conference Short Course: Radiation Effects in the Space Environment, IEEE, Norfolk, Virginia, 1999.

3. S.M. Seltzer, Predictions of Dose from Electrons in Space, NASAVCR-191617, December, 1992.

4. J. Barth, "Modeling Space Radiation Environments", in the 1997 IEEE NSREC. IEEE Nuclear and Space Radiation Effects Conference Short Course: Applying Computer Simulation Tools to Radiation Effects Problems, IEEE, Snowmass Village, Colorado, 1997.

5. D.M. Sawyer and J.I. Vette, AP.8 Trapped Proton Environment for Solar Maximum and Solar Minimum, NSSDC, Report 76-06, Greenbelt, Maryland, 1976. 
6. J.I. Vette, "AE/AP Trapped Particle Flux Maps 1966 - 1980", available on WWW at: http://nssdc.gsfc.nasa.gov/space/model/magnetos/aeap.html (updated 1996), 2000.

7. G.F. Knoll, Radiation Detection and Measurement, Second Edition. John Wiley \& Sons, 1989.

8. T.W. Armstrong and B.L. Colborn, TRAP/SEE Code Users Manual for Predicting Trapped Radiation Environments, NASA/CR-2000-209879, NASA/MSFC, January, 2000.

9. S.L. Huston and K.A. Pfitzer, Space Environment Effects: Low-Altitude Trapped Radiation Model, NASA/CR-1998-208593, NASA/MSFC, August, 1998.

10. M.S. Gussenhoven, et al., "APEXRAD: Low Altitude Orbit Dose as a Function of Inclination, Magnetic Activity, and Solar Cycle", IEEE Trans. on Nuclear Sci.. 44, 2161 - 2173, 1997.

11. S.M. Seltzer. Electron, "Electron Bremsstrahlung, and Proton Depth-Dose Data for SpaceShielding Applications", IEEE Trans. Nuclear Sci., NS-26, 4896. 1979.

12. A.R. Fredrickson, P.B. McGrath, and P. Leung. "Radiation-Induced Electrical Insulation Breakdown". in Conference on Electrical Insulation and Dielectric Phenomena, 210 - 217. 1989.

13. D.A. Russell, L.B. Fogdall, and G. Bohnhoff-Hlavacek, Simulated Space Environmental Testing on Thin Films, NASA/CR-2000-210101. NASA/Langley, April, 2000.

14. R.A. Reed, K. LaBel, H. Kim, H. Leidecker, and J. Lohr, "Test Report of Proton and Neutron Exposures of Devices that Utilize Optical Components and are Contained in the CIRS Instrument", unpublished report, available on WWW at: http://radhome.gsfc.nasa.gov/radhome/papers/1090397.html, 15 January 1998.

15. J.F. Ziegler, J.P. Biersack, and V. Littmark, The Stopping Range of lons in Solids, Pergamon Press, New York, 1985. 